Tôhoku Math. Journ.

28 (1976), 293-303.

\title{
SOME CONDITIONS ON A REDUCTIVE OPERATOR IMPLYING NORMALITY OR SPECTRALITY
}

\author{
KOH-ICHI KITANO
}

(Received July 31, 1975)

In this note, we shall show some sufficient conditions that a reductive operator acting in a complex Hilbert space be normal or spectral. It is a generalization of some results on operators with reducing invariant subspaces in [9]. In $\S 2$, if a reductive operator $T$ is the sum of a normal operator, whose spectrum lies on a $C^{2}$-Jordan curve, and a compact operator which belongs to the Macaev class $\mathscr{C}_{\omega}$, then $T$ is normal. In $\S 3$, if the spectrum of a reductive operator $T$ lies on a $C^{2}$-Jordan curve $J$ and if the resolvent of $T$ satisfies the growth condition (C) near $J$, then $T$ is spectral.

Throughout this note $\mathscr{H}$ will be a (not necessarily separable) complex Hilbert space. We denote by $\mathscr{B}(\mathscr{H})$ the algebra of all bounded linear operators acting in $\mathscr{H}$. We say that the bounded linear operator $T$ is reductive if every invariant subspace of $T$ reduces $T$. A well-known unsolved problem is "must a reductive operator be normal?". It has recently been shown that this problem is equivalent to the invariant subspace conjecture [5]. The answer is affirmative for compact operators [1] and for polynomially compact operators [11], [10] and in [9] has been shown that if $T-T^{*}$ or $I-T^{*} T$ belongs to a von Neumann-Schatten class $\mathscr{C}_{p}(1 \leqq p<\infty)$ or if $T$ is similar to the sum of a normal operator and a commuting compact operator, then $T$ is reductive only if $T$ is normal, and if the spectrum of a reductive operator $T$ lies on a $C^{2}$-Jordan curve and if the resolvent of $T$ satisfies an exponential growth condition, then $T$ is spectral.

In this note we always represent $J$ by the canonic form $J=\{g(s)$ : $s \in[0, l]\}$, where $s$ denotes the length of arc (if $J$ is a Jordan curve we have $g(0)=g(l))$. The set of all operators $T \in \mathscr{B}(\mathscr{H})$ such that the spectrum of $T$ lies on a $C^{2}$-Jordan curve $J$ will be denoted by $\mathscr{B}(\mathscr{H}, J)$. We denote by $\sigma(T)$ and $\rho(T)$, the spectrum and the resolvent set of $T$, respectively. For any complex number $\lambda$ and a subset $E$ of a complex plane $C$, we put $d(\lambda, E)=\inf \{|\lambda-z|: z \in E\}$. Let $\mathscr{X}$ be an invariant subspace of $T$, we denote by $T \mid \mathscr{Z}$ the restriction of $T$ to $\mathscr{X}$. 
1. Preliminaries. In this section we note some known results and preliminary lemmas for later use.

Let $T \in \mathscr{B}(\mathscr{H}, J)$.

Definition 1.1. ([7]) We say that the resolvent of $T$ has the growth condition (C) near $J$ if

$$
\int_{0}^{\varepsilon} \log \log M(\delta) d \delta<\infty
$$

for some sufficiently small $\varepsilon>0$, where $M(\delta)=\sup \left\{\left\|(\lambda I-T)^{-1}\right\|: d(\lambda, J)>\delta\right\}$.

In [9], the resolvent is said to have an exponential growth condition, if $d(\lambda, J)^{p} \log \left\|(\lambda I-T)^{-1}\right\|, \lambda \notin J$ is bounded near $J$ for some $p \geqq 1$. If the resolvent has an exponential growth condition near $J$, then it has the growth condition (C) near $J$.

LEMma 1.2. Let $T \in \mathscr{B}(\mathscr{H}, J)$. Suppose that the resolvent of $T$ satisfies the growth condition $(C)$ near $J$. If $J_{0}$ is an open subarc of $J$ and if we define by

$$
\begin{aligned}
& \mathscr{S}=\left\{x \in \mathscr{H}:(\lambda I-T)^{-1} x\right. \text { has an analytic } \\
& \text { continuation to the complement of } \left.\bar{J}_{0}\right\} \text {, }
\end{aligned}
$$

(bar denote the closure), then $\mathscr{S}$ is a closed hyperinvariant subspace of $T$.

Moreover, if $\sigma(T) \cap J_{0} \neq \varnothing$, then $\mathscr{S} \neq\{0\}$ and $\sigma(T \mid \mathscr{S}) \subset \bar{J}_{0}$. If $\sigma(T)$ consists of more than one point, then we can choose an open subarc $J_{0}$ of $J$ such that $\mathscr{S}$ is a proper subspace.

Proof. In Lemma 2.4 of [7], we have shown that $\mathscr{S}$ is a closed invariant subspace of $T$. Moreover, taking into consideration of Lemmas 2.4 and 2.5 in [7], if $\sigma(T) \cap \bar{J}_{0} \neq \varnothing$, then $\mathscr{S} \neq\{0\}$ and if $\sigma(T)$ contains more than one point, we can choose an open subarc $J_{0}$ of $J$ such that $\mathscr{S}$ is a proper subspace.

For the proof of this lemma, next we show that $\mathscr{S}$ is a hyperinvariant subspace of $T$. If $S \in \mathscr{B}(\mathscr{H})$, which commutes with $T$, then $S$ commutes with the resolvent, $(\lambda I-T)^{-1}$, of $T$ for every $\lambda$ in $\rho(T)$. Now, let $R(\lambda, T: x)$ denote the analytic continuation of $(\lambda I-T)^{-1} x$, from the equation

$$
(\lambda I-T)^{-1} S x=S(\lambda I-T)^{-1} x
$$

for every $x \in \mathscr{S}$, then it is clear that $\operatorname{SR}(\lambda, T: x)$ is an analytic continuation of $(\lambda I-T)^{-1} S x$. Therefore $S x \in \mathscr{S}$ for every $x \in \mathscr{S}$, i.e., $\mathscr{S}$ is hyperinvariant subspace of $T$.

To complete the proof we have only to show that $\sigma(T \mid \mathscr{S}) \subset \bar{J}_{0}$ for 
the case of $\sigma(T) \cap J_{0} \neq \varnothing$. In the following, we can use an argument of (p. 201, [3]). We first show that $R(\lambda, T: x) \in \mathscr{S}$ for every $x \in \mathscr{S}$. For a fixed $\lambda \in C \backslash \bar{J}_{0}$, we define the function $G_{\lambda}(\mu)$ such that

$$
G_{\lambda}(\mu)= \begin{cases}-\frac{R(\mu, T: x)-R(\lambda, T: x)}{\mu-\lambda} & \text { if } \mu \neq \lambda, \\ -R^{\prime}(\lambda, T: x) & \text { if } \mu=\lambda,\end{cases}
$$

then $G_{\lambda}(\mu)$ is analytic in $C \backslash \bar{J}_{0}$ and follows that

$$
\begin{aligned}
(\mu I-T) G_{\lambda}(\mu)= & -(\mu I-T)\left\{\frac{R(\mu, T: x)-R(\lambda, T: x)}{\mu-\lambda}\right\} \\
= & -(\mu-\lambda)^{-1}\{(\mu I-T) R(\mu, T: x)-(\lambda I-T) R(\lambda, T: x) \\
& -(\mu-\lambda) R(\lambda, T: x)\} \\
= & -(\mu-\lambda)^{-1}\{x-x-(\mu-\lambda) R(\lambda, T: x)\}=R(\lambda, T: x),
\end{aligned}
$$

for $\mu \neq \lambda$. If we make $\mu \rightarrow \lambda$, we obtain that the equality between the extreme terms of the above relation of equalities holds also for $\mu=\lambda$. Therefore if $x \in \mathscr{S}$, then $R(\lambda, T: x) \in \mathscr{S}$.

Next, let $\lambda_{0} \in C \backslash \bar{J}_{0}$. We define the operator $H$ acting in $\mathscr{S}$ by the following

$$
H x=R\left(\lambda_{0}, T: x\right) \text {. }
$$

It is evident that $H$ is linear. We will show that $H$ is closed. Let $\left\{x_{n}\right\} \subset \mathscr{S}$ with $\lim _{n \rightarrow \infty} x_{n}=x$ and $\lim _{n \rightarrow \infty} H x_{n}=y$. From the fact that $\mathscr{S}$ is closed it follows that $x \in \mathscr{S}$ and $y=\lim _{n \rightarrow \infty} H x_{n}=\lim _{n \rightarrow \infty} R\left(\lambda_{0}, T: x_{n}\right) \in \mathscr{S}$. We have

$$
\left(\lambda_{0} I-T\right) H x_{n}=\left(\lambda_{0}-T\right) R\left(\lambda_{0}, T: x_{n}\right)=x_{n},
$$

from which follows $\left(\lambda_{0} I-T\right) y=x$. While,

$$
\left(\lambda_{0} I-T\right) R\left(\lambda_{0}, T: x\right)=x,
$$

therefore we have

$$
\left(\lambda_{0} I-T\right)\left\{y-R\left(\lambda_{0}, T: x\right)\right\}=0 .
$$

Let us now put $z=y-R\left(\lambda_{0}, T: x\right)$. Having $y, R\left(\lambda_{0}, T: x\right) \in \mathscr{S}$, it follows that $z \in \mathscr{S}$. Thus $(\lambda I-T)^{-1} z$ has an analytic continuation to the complement of $\bar{J}_{0}$. Let the function $h$ from $C \backslash\left\{\lambda_{0}\right\}$ into $\mathscr{H}$ be defined by

$$
h(\lambda)=\left(\lambda-\lambda_{0}\right)^{-1} z \text {. }
$$

Then we have

$$
\begin{aligned}
(\lambda I-T) h(\lambda) & =\left(\lambda-\lambda_{0}\right)^{-1}(\lambda I-T) z \\
& =\left(\lambda-\lambda_{0}\right)^{-1}\left\{\left(\lambda_{0} I-T\right) z+\left(\lambda-\lambda_{0}\right) z\right\}=z .
\end{aligned}
$$


Consequently it results that $R(\lambda, T: z)$ is an entire function. Since it satisfies

$$
(\lambda I-T) R(\lambda, T: z)=z
$$

for $|\lambda|>\|T\|$. We have

$$
R(\lambda, T: z)=(\lambda I-T)^{-1} z \rightarrow 0 \quad(|\lambda| \rightarrow \infty),
$$

therefore by Liouville's theorem $R(\lambda, T: z) \equiv 0$. Thus we have

$$
z=\frac{1}{2 \pi i} \int_{\Gamma} R(\lambda, T: z) d \lambda=0,
$$

where $\Gamma=\{\lambda:|\lambda|=\|T\|+1\}$, therefore $y=R\left(\lambda_{0}, T: x\right)=H x$. It follows that the operator $H$ is closed and, therefore according to Banach's closed graph theorem (see, p. 79 in [15]), $H \in \mathscr{B}(\mathscr{S})$. We observe that

$$
\left(\lambda_{0} I-T\right) H x=\left(\lambda_{0} I-T\right) R\left(\lambda_{0}, T: x\right)=x
$$

for every $x \in \mathscr{S}$, therefore we have

$$
\text { (*) } \quad\left(\lambda_{0} I|\mathscr{S}-T| \mathscr{S}\right) H=I \mid \mathscr{S} \text {. }
$$

In the next place for every $x \in \mathscr{S}$, we have

$$
R\left(\lambda_{0}, T:\left(\lambda_{0} I-T\right) x\right)=\left(\lambda_{0} I-T\right) R\left(\lambda_{0}, T: x\right),
$$

from which, according to the definition of $H$, follows that

$$
H\left(\lambda_{0} I-T\right) x=R\left(\lambda_{0}, T:\left(\lambda_{0} I-T\right) x\right)=x,
$$

therefore we have

$$
H\left(\lambda_{0} I|\mathscr{S}-T| \mathscr{S}\right)=I \mid \mathscr{S} .
$$

From (*), (**) follows that $H=\left(\lambda_{0} I|\mathscr{S}-T| \mathscr{S}\right)^{-1}$, therefore we have $\sigma(T \mid \mathscr{S}) \subset \bar{J}_{0}$. The proof of Lemma 1.2 is now completed.

The following lemma is found in [7].

LeMma 1.3. Let $T \in \mathscr{B}(\mathscr{H})$. Write $T=A+B$, where $T$ is the sum of a normal operator $A$, whose spectrum lies on $J$, and a compact operator $B$ which belongs to the Macaev class $\mathscr{C}_{\omega}$. Then any point $\lambda$ with $\lambda \notin \sigma(A)$ is either $\lambda \in \rho(T)$ or an eigenvalue with finite multiplicity of $T$.

REMARK. The Macaev class $\mathscr{C}_{\omega}$ is the set of compact operators for which $\|T\|_{\omega}=\sum_{n} \mu_{n} / n<\infty$, where $\mu_{n}(n=1,2,3, \cdots)$ are the eigenvalues of $\left(T^{*} T\right)^{1 / 2}$. It is easy to see that $\mathscr{C}_{\omega}$ contains all von Neumann-Schatten classes $\mathscr{C}_{p}(1 \leqq p<\infty)$ of compact operators (cf. $\S 3$ in [7] or [8]).

The next lemma was established in the proof of Theorem 3.1 in [7]. 
Lemma 1.4. Let $T$ be as above (in Lemma 1.3). Suppose further that the spectrum of $A$ does not separate the complex plane $\boldsymbol{C}$ and the point spectrum of $T$ is empty. Then the resolvent of $T$ satisfies the growth condition $(C)$ near $J$.

The next lemma is a trivial generalization of Lemma 2.3 in [6], (see, Lemma 2.5 in [9]).

Lemma 1.5. If $T$ is a quasi-nilpotent operator and $T^{*} T-T T^{*}$ is a compact operator, then $T$ is compact.

The following lemma was established in [9].

Lemma 1.6. Let $T \in \mathscr{B}(\mathscr{H})$. If $\left\{\mathscr{N}_{\alpha}\right\}$ is a family of reducing subspaces for $T$ such that $T \mid \mathscr{K}_{\alpha}$ is normal for each $\alpha$, then $T \mid\left(\vee\left\{\mathscr{K}_{\alpha}\right\}\right)$ is normal, where $\vee\left\{\mathscr{K}_{\alpha}\right\}$ denotes the closed linear span of the family $\left\{\mathscr{L}_{\alpha}\right\}$.

Lemma 1.7. Suppose $K=T^{*} T-T T^{*}$ is a compact operator, and let $\left\{\mathscr{L}_{\alpha}\right\}$ be a totally ordered (by inclusion relation) family of subspaces each of which reduces $T$ and satisfies $\left\|K \mid \mathscr{M}_{\alpha}\right\|=\|K\|$. Then $\left\|K \mid \mathscr{K}_{0}\right\|=$ $\|K\|$, where $\mathscr{K}_{0}=\cap \mathscr{N}_{\alpha}$.

Proof. The proof is quite similar to one of Lemma 2 in [11]. Let $\varepsilon>0$ be given. For each $\alpha$, there exists a unit vector $x_{\alpha}$ in $\mathscr{H}$ such that

$$
\left\|\left(P_{0}-P_{\alpha}\right) K x_{\alpha}\right\|>\left\|\left(P_{0}-P_{\alpha}\right) K\right\|-\varepsilon / 4,
$$

where $P_{\alpha}, P_{0}$ are orthogonal projections on $\mathscr{N}_{\alpha}, \mathscr{A}_{0}$, respectively. Since $\left\{x_{\alpha}\right\}$ is a bounded set and $K$ is a compact operator, we can choose a subset $\left\{x_{\alpha_{\nu}}\right\}$ such that $\left\{K x_{\alpha_{\nu}}\right\}$ converges strongly to a certain vector $x$. Since $P_{0}$ is a strong limit of $P_{\alpha}$, there exists a $\nu$ such that

$$
\left\|\left(P_{\alpha_{\nu}}-P_{0}\right) x\right\|<\varepsilon / 4,\left\|K x_{\alpha_{\nu}}-x\right\|<\varepsilon / 4 .
$$

Combining above inequalities we have

$$
\left\|\left(P_{0}-P_{\alpha_{\nu}}\right) K\right\|<\varepsilon .
$$

Since $\varepsilon>0$ is arbitrary, we have

$$
\left\|K \mid \mathscr{M}_{0}\right\|=\left\|P_{0} K\right\|=\lim \left\|P_{\alpha_{\nu}} K\right\|=\|K\| \text {. }
$$

The proof of Lemma 1.7 is now completed.

2. Some sufficiently conditions on a reductive operator implying normality. In this section we shall see the following theorem to be valid.

THEOREM 2.1. If $T$ is a reductive operator, if $T=A+B$, where $T$ is the sum of a normal operator $A$, whose spectrum lies on $J$ (the spectrum 
of $A$ does not separate the complex plane) and a compact operator $B$ which belongs to the Macaev class $\mathscr{C}_{\omega}$, then $T$ is normal.

As a immediate consequence of this theorem, we have the following corollary.

COROLLARY 2.2. If $T$ is a reductive operator and if the imaginary part of $T$ is in the Macaev class $\mathscr{C}_{\omega}$, then $T$ is normal.

Proof of Theorem 2.1. For each point $\lambda \in \sigma_{p}(T)$ (the point spectrum of $T)$, set $\mathscr{N}(\lambda)=\{x \in \mathscr{H}: T x=\lambda x\}$. Since $\mathscr{N}(\lambda)$ is an invariant subspace of $T$, each $\mathscr{N}(\lambda), \lambda \in \sigma_{p}(T)$ reduces $T$ by assumption, we have $T T^{*} x=\lambda T^{*} x$ for $x \in \mathscr{N}(\lambda)$, it follows $\left\|T^{*} x-\bar{\lambda} x\right\|^{2}=0$, therefore $T^{*} x=$ $\bar{\lambda} x$ for $x \in \mathscr{N}(\lambda)$. Thus the restriction of $T$ onto $\mathscr{N}=\vee\{\mathscr{N}(\lambda): \lambda \in$ $\left.\sigma_{p}(T)\right\}$ is a normal operator (by Lemma 1.6). We must show that the restriction of $T$ to the reducing subspace $\mathscr{N}^{\perp}$ is also normal. Clearly by Lemma $1.3 \sigma\left(T \mid \mathscr{N}^{\perp}\right) \subset J$ and Lemma 1.4 implies that the resolvent of $T \mid \mathscr{N}^{\perp}$ satisfies the growth condition (C) near $J$.

We shall break the proof into portions. Hereafter to simplify we suppose that $\sigma(T) \subset J$ and the resolvent of $T$ satisfies the growth condition (C) near $J$.

(i) Every non-zero reducing subspace of $T$ includes a proper invariant subspace of $T$ or else is one-dimensional.

Let $\mathscr{M}$ be a reducing subspace of $T$. Since $\sigma(T \mid \mathscr{C}) \subset \sigma(T)$, the resolvent of $T \mid \mathscr{M}$ satisfies the same growth condition as the resolvent of $T$ does, consequently the resolvent of $T \mid \mathscr{M}$ satisfies the growth condition (C) near $J$. In the first place if $\sigma(T \mid \mathscr{M})$ consists of more than one-point. From Lemma 1.2, there exists a proper invariant subspace of $T \mid \mathscr{M}$, thus it also a proper invariant subspace of $T$. The next, if $\sigma(T \mid \mathscr{M})$ consists of a single point, say $\{\lambda\}$. Since $\mathscr{M}$ is a reducing subspace of $T,(T \mid \mathscr{C})^{*}=T^{*} \mid \mathscr{L}$, we have

$$
(\lambda I-T)^{*}(\lambda I-T)-(\lambda I-T)(\lambda I-T)^{*}=T^{*} T-T T^{*} .
$$

On the other hand, $A$ is normal and $B$ is compact, it follows that $T^{*} T-$ $T T^{*}$ is compact. According to Lemma 1.5, $(\lambda I-T) \mid \mathscr{C}$ is compact. Hence $\mathscr{C}$ is a one-dimensional or $(\lambda I-T) \mid \mathscr{C}$ has a proper invariant subspace by Aronszajn-Smith theorem [2]. It follows from above arguments that the only minimal invariant subspace of $T$ are one-dimensional and reducing.

(ii) Let $\mathscr{N}_{0}$ be the closed linear span of the one-dimensional reducing subspaces of $T$ and let $K=T^{*} T-T T^{*}$. Then $\mathscr{N}_{0}$ reduces $T$ and $K$. According to Lemma 1.6, $T \mid \mathscr{N}_{0}$ is normal, thus to complete the proof 
we have to show that $T \mid \mathscr{N}_{0}^{\perp}$ is normal, it suffices to prove that $K \mid \mathscr{N}_{0}^{\perp}=0$.

(iii) Next we shall show that $K \mid \mathscr{N}_{0}{ }^{\perp}=0$. Since $\mathscr{N}_{0}^{\perp}$ is reducing subspace of $T$, we have $\sigma\left(T \mid \mathscr{N}_{0}{ }^{\perp}\right) \subset \sigma(T) \subset J$. Therefore the resolvent of $T \mid \mathscr{N}_{0}{ }^{\perp}$ satisfies the growth condition (C) near $J$. From the result in (i), every non-zero reducing subspace of $T \mid \mathscr{N}_{0}^{\perp}$ includes a proper invariant subspace of $T \mid \mathscr{N}_{0}{ }^{\perp}$ or else is one-dimensional. Now we suppose to be $K \mid \mathscr{N}_{0}{ }^{\perp} \neq 0$. Let $\mathscr{F}$ be the family of subspaces $\mathscr{M} \subset \mathscr{N}_{0}{ }^{\perp}$ such that reduce $T \mid \mathscr{N}_{0}{ }^{\perp}$ and satisfy the condition $\left\|P_{\mathscr{M}} K\left|\mathscr{N}_{0}{ }^{\perp}\|=\| K\right| \mathscr{N}_{0}{ }^{\perp}\right\|$, where $\left\{P_{\mathscr{A}}\right\}$ are the orthogonal projections on $\mathscr{A}$. By Lemma 1.7, $\mathscr{F}$ is inductive, therefore Zorn's lemma implies that $\mathscr{F}$ contains a minimal member $\mathscr{M}_{0}$. On account of $K \mid \mathscr{N}_{0}^{\perp} \neq 0$ (by assumption), $\mathscr{A}_{0} \neq\{0\}$, if $\operatorname{dim} \mathscr{K}_{0} \geqq 2$, then $\mathscr{K}_{0}$ contains a proper invariant subspace $\mathscr{K}_{1}$ (by Lemma 1.2). By hypothesis of this theorem $\mathscr{K}_{1}$ reduces $T \mid \mathscr{N}_{0}^{\perp}$ and we have

$$
\begin{aligned}
\left\|K \mid \mathscr{N}_{0}{ }^{\perp}\right\| & =\left\|P_{\mathscr{M}_{0}} K \mid \mathscr{N}_{0}{ }^{\perp}\right\| \\
& =\max \left\{\left\|P_{\mathscr{M}_{1}} K\left|\mathscr{N}_{0}{ }^{\perp}\|,\|\left(P_{\mathscr{N}_{0}}-P_{\mathscr{L}_{1}}\right) K\right| \mathscr{N}_{0}{ }^{\perp}\right\|\right\},
\end{aligned}
$$

where $P_{\mathscr{N}_{0}}, P_{\mathscr{M}_{1}}$ are the orthogonal projections on $\mathscr{M}_{0}$ and $\mathscr{M}_{1}$ respectively. It follows that $\mathscr{M}_{1}$ or $\mathscr{M}_{0} \cap \mathscr{M}_{1}{ }^{\perp}$ is a member of $\mathscr{F}$. This contradicts the minimality of $\mathscr{H}_{0}$, for both $\mathscr{M}_{1}$ and $\mathscr{K}_{0} \cap \mathscr{M}_{1}^{\lrcorner}$are properly contained in $\mathscr{K}_{0}$. Thus $\operatorname{dim} \mathscr{K}_{0}=1$, i.e., there exists a one-dimensional reducing subspace of $T \mid \mathscr{N}_{0}{ }^{\perp}$. Again this contradicts the construction of $\mathscr{N}_{0}$, therefore $K \mid \mathscr{N}_{0}{ }^{\perp}=0$, i.e., $T \mid \mathscr{N}_{0}{ }^{\perp}$ is normal. The proof of Theorem 2.1 is now completed.

\section{A sufficient condition on a reductive operator implying spectrality.} In this section we shall show that if a reductive operator belongs to $\mathscr{B}(\mathscr{H}, J)$ whose resolvent satisfies the growth condition (C) near $J$ is spectral, (in fact, is the sum of a normal operator and a commuting quasinilpotent operator). The same result has been obtained in [9] for an exponential growth condition, and some related results have also been obtained by J. G. Stampfli [13], [14]. The idea of the proof is to construct a spectral measure locally by means of techniques similar to those used by J. T. Schwartz in [12]. The arguments in this section is quite similar to that of [9].

The following lemma was established in [9].

LEMma 3.1. Suppose T has closed hyperinvariant reducing subspaces $\mathscr{A}_{1}, \mathscr{A}_{2}, \cdots, \mathscr{M}_{k}$ such that, for each $i, T \mid \mathscr{A}_{i}=A_{i}+B_{i}$ where $A_{i}$ is normal on $\mathscr{M}_{i}, B_{i}$ is quasi-nilpotent on $\mathscr{M}_{i}$ and $A_{i}$ commutes with $B_{i}$. If the $\mathscr{K}_{i}(i=1,2, \cdots, k)$ span $\mathscr{H}$, then $T=A+B$, where $A$ is normal 
and $B$ is quasi-nilpotent such that $A$ commutes with $B$.

Lemma 3.2. Let $T \in \mathscr{B}(\mathscr{H}, J)$ be a reductive operator. Suppose that the resolvent $T$ satisfies the growth condition $(C)$ near $J$. If $\mathscr{S}$ is an invariant subspace in Lemma 1.2 , then $T \mid \mathscr{S}=A+B$, where $A$ is normal, $B$ is quasi-nilpotent and $A$ commutes with $B$.

Proof. Let $J_{0}=\{g(s): s \in(a, b)\}$, (take note of $J=\{g(s): s \in[0, l]\}$ ). Then $0<a<b<l$. By Lemma 1.2, $\mathscr{S}$ is a closed hyperinvariant subspace for $T$. Choose $c$ and $d$ such that $0<c<a<b<d<l$, and for $t \in(c, d)$ now we define $\mathscr{S}_{t}$ by

$$
\begin{aligned}
\mathscr{S}_{t}=\{x \in \mathscr{H}: & (\lambda I-T)^{-1} x \text { has an analytic continuation } \\
& \text { to the complement of }\{g(s): s \in[c, t]\}\} .
\end{aligned}
$$

Then each $\mathscr{S}_{t}$ is a closed hyperinvariant subspace for $T$, and $\sigma\left(T \mid \mathscr{S}_{t}\right) \subset$ $\{g(s): s \in[c, t]\}$, (by Lemma 1.2). For each $t \in(c, d)$, let $P_{t}$ denote the orthogonal projection onto $\mathscr{S}_{t}$, since $T$ is reductive, it follows that every $P_{t}$ commute with $T$. Now $t_{1}<t_{2}$ implies $\mathscr{S}_{t_{1}} \subset \mathscr{S}_{t_{2}}$, or $P_{t_{1}} \leqq P_{t_{2}}$. Next we define the normal operator $A$ by

$$
A=\int_{a}^{b} g(t) d P_{t} .
$$

Now $\mathscr{S}$ is invariant under every operator commuting with $T$, and is thus invariant under each $P_{t}$ and hence reduces $A$.

The desired conclusion will therefore be established as soon as it is shown that $(T \mid \mathscr{S})-(A \mid \mathscr{S})$ is quasi-nilpotent. For this we first show that $t_{1}<t_{2}$ implies that

$$
\sigma\left(T \mid\left(\mathscr{S}_{t_{2}} \ominus \mathscr{S}_{t_{1}}\right)\right) \subset\left\{g(s): s \in\left[t_{1}, t_{2}\right]\right\} .
$$

We know by Lemma 1.2 that

$$
\sigma\left(T \mid\left(\mathscr{S}_{t_{2}}\right)\right) \subset\left\{g(s): s \in\left[c, t_{2}\right]\right\},
$$

so we need only show that $s_{0} \in\left[c, t_{1}\right)$ implies

$$
g\left(s_{0}\right) \notin \sigma\left(T \mid\left(\mathscr{S}_{t_{2}} \ominus \mathscr{S}_{t_{1}}\right)\right) .
$$

We put $T_{1}=T \mid\left(\mathscr{S}_{t_{2}} \ominus \mathscr{S}_{t_{1}}\right)$. Note that

$$
\left(\lambda I-T_{1}\right)^{-1}=(\lambda I-T)^{-1} \mid\left(\mathscr{S}_{t_{2}} \ominus \mathscr{S}_{t_{1}}\right)
$$

for $\lambda \notin \sigma(T)$. If $s_{0} \in\left[c, t_{1}\right)$, let $J_{1}$ be an open subarc of $J$ contained in $\left\{g(s): s \in\left[c, t_{1}\right)\right\}$ and containing $g\left(s_{0}\right)$. Since $\left(\lambda I-T_{1}\right)^{-1}$ satisfies the same growth condition as $(\lambda I-T)^{-1}$ does, i.e., $\left(\lambda I-T_{1}\right)^{-1}$ satisfies the growth condition (C) near $J$, therefore Lemma 1.2 implies that the space 


$$
\begin{gathered}
\mathscr{C}=\left\{x \in \mathscr{S}_{t_{2}} \ominus \mathscr{S}_{t_{1}}:\left(\lambda I-T_{1}\right)^{-1} x\right. \text { has an analytic continuation } \\
\text { to the complement of } \left.\bar{J}_{1}\right\},
\end{gathered}
$$

is a closed hyperinvariant subspace of $T_{1}$. But if $x \in \mathscr{L}$, then since $(\lambda I-T)^{-1} x=\left(\lambda I-T_{1}\right)^{-1} x$ for $\lambda \notin \sigma(T)$, implies that $x \in \mathscr{S}_{t_{1}}$. Hence $\mathscr{M}=$ $\{0\}$, and from Lemma 1.2 implies $J_{1} \cap \sigma\left(T_{1}\right)=\varnothing$. Thus $g\left(s_{0}\right) \notin \sigma\left(T_{1}\right)$ and $\sigma\left(T \mid\left(\mathscr{S}_{t_{2}} \ominus \mathscr{S}_{t_{1}}\right)\right) \subset\left\{g(s): s \in\left[t_{1}, t_{2}\right]\right\}$. We can now use an argument of Dunford's (p. 1940 in Part III of [4]) to show that $(T \mid \mathscr{S})-(A \mid \mathscr{S})$ is a quasinilpotent operator.

To prove this, given any $\varepsilon>0$ form a partition

$$
a=t_{0}<t_{1}<t_{2}<\cdots<t_{n}=b
$$

of $[a, b]$ such that every diameter of $\operatorname{subarc}\left\{g(s): s \in\left[t_{i-1}, t_{i}\right]\right\}(i=1,2, \cdots$, $n$ ) less than $\varepsilon$. Then the spectrum of operators

$$
T_{i}=T\left|\left(\mathscr{S}_{t_{i}} \ominus \mathscr{S}_{t_{i-1}}\right), A_{i}=A\right|\left(\mathscr{S}_{t_{i}} \ominus \mathscr{S}_{t_{i-1}}\right)
$$

are each contained in $\left\{g(s): s \in\left[t_{i-1}, t_{i}\right]\right\}$ and, since $T_{i}$ and $A_{i}$ commute, the spectrum of $T_{i}-A_{i}$ is contained in $\left\{g\left(s_{1}\right)-g\left(s_{2}\right): s_{1}\right.$ and $\left.s_{2} \in\left[t_{i-1}, t_{i}\right]\right\}$. Thus the spectral radius of $T_{i}-A_{i}$ is at most $2 \varepsilon$. Now we define the space by

$$
\begin{aligned}
\mathscr{S}= & {\left[\mathscr{S} \cap \mathscr{S}_{t_{0}}\right] \oplus\left[\mathscr{S} \cap\left(\mathscr{S}_{t_{1}} \ominus \mathscr{S}_{t_{0}}\right)\right] \oplus\left[\mathscr{S} \cap\left(\mathscr{S}_{t_{2}} \ominus \mathscr{S}_{t_{1}}\right)\right] \oplus \cdots } \\
& \oplus\left[\mathscr{S} \cap\left(\mathscr{S}_{t_{n}} \ominus \mathscr{S}_{t_{n-1}}\right)\right] .
\end{aligned}
$$

Let $T_{0}$ and $A_{0}$ respectively denote the restriction of $T$ and $A$ to $\mathscr{S} \cap \mathscr{S}_{t_{0}}$. (Note that $\mathscr{S} \cap \mathscr{S}_{t_{0}}$ could be $\{0\}$ in which case this term is unnecessary.) Then $\sigma\left(T_{0}\right)=\sigma\left(A_{0}\right)=\left\{g\left(t_{0}\right)\right\}$, and $\sigma\left(T_{0}-A_{0}\right)=\{0\}$. Now

$$
(T-A) \mid \mathscr{S}=\sum_{i=0}^{n} \oplus P\left(T_{i}-A_{i}\right),
$$

where $P$ is the orthogonal projection onto $\mathscr{S}$, and since the spectrum of the direct sum of finite operators is the union of their spectrum, it follows that the spectral radius of $(T-A) \mid \mathscr{S}$ is at most $2 \varepsilon$. Since $\varepsilon$ is an arbitrary positive number, it results that $\sigma((T-A) \mid \mathscr{S})=\{0\}$. Thus $(T \mid \mathscr{S})-(A \mid \mathscr{S})$ is a quasi-nilpotent operator. The proof of Lemma 3.2 is now completed.

THEOREM 3.3. Let $T \in \mathscr{B}(\mathscr{H}, J)$ be a reductive operator. Suppose that the resolvent of $T$ satisfies the growth condition $(C)$ near $J$. Then $T=A+B$, where $A$ is normal, $B$ is quasi-nilpotent and $A$ commutes with $B$, i.e., $T$ is spectral.

Proof. We can choose an open subarc $J_{0}$ of $J$ such that the corresponding closed hyperinvariant subspace $\mathscr{S}$ given by Lemma 1.2 is not 
zero. According to Lemma 3.2, T|S $\mathscr{S}$ is the sum of a normal operator and a commuting quasi-nilpotent operator. Thus by Lemma 3.1, we need only show that the whole space $\mathscr{H}$ is the closed linear span of finitely many such subspaces $\mathscr{S}$. Since $\sigma(T)$ is compact, there exists a finite collection $\mathscr{E}$ of arcs such as $J_{0}$ above whose union includes $\sigma(T)$. Let $\mathscr{L}_{0}$ be the closed linear span of the corresponding subspaces $\mathscr{S}$. Next, we must show that $\mathscr{K}_{0}^{\perp}=\{0\}$. If not, then let $\lambda_{0} \in \sigma\left(T \mid \mathscr{L}_{0}^{\perp}\right)$ and consider the arc $J_{0}$ in $\mathscr{E}$ such that $\lambda_{0} \in J_{0}$. Then the closed invariant subspace for $T \mid \mathscr{C}_{0}^{\perp}$ corresponding to $J_{0}$ given by Lemma 1.2 would contain a non-zero vector $x$. Since $\mathscr{A}_{0}^{\perp}$ reduces $T$, we have

$$
\left(\lambda I-T \mid \mathscr{K}_{0}^{\perp}\right)^{-1} x=(\lambda I-T)^{-1} x,
$$

and thus $(\lambda I-T)^{-1} x$ would have an analytic continuation to the complement of $\bar{J}_{0}$. Hence $x \in \mathscr{S}$, when $\mathscr{S}$ is the invariant subspace for $T$ corresponding to $J_{0}$. But $\mathscr{S} \subset \mathscr{K}_{0}$, contradicting the fact that $x \in \mathscr{K}_{0}{ }^{\perp}$, it results that $\mathscr{C}_{0}^{\perp}=\{0\}$. Thus $\mathscr{C}_{0}=\mathscr{C}$ and the conclusion of this theorem follows from Lemma 3.1.

\section{REFERENCES}

[1] T. ANDô, Note on invariant subspaces of a compact normal operator, Arch. der Math., 14 (1963), 337-340.

[2] N. ARonszajn and K. T. Smith, Invariant subspaces of completely continuous operators, Ann. of Math., II Ser., 60 (1954), 345-350.

[3] I. Colojoara, Elemente de Teorie Spectrala, Editura Academiei Republicii Socialiste Romania, Bucuresti, 1968.

[4] N. Dunford and J. T. Schwartz, Linear Operators, Part I, Part II, Part III, Interscience, New York, 1958, 1963, 1971.

[5] J. A. Dyer, E. A. Pedersen and P. Porcelli, An equivalent formulation of the invariant subspace conjecture, Bull. Amer. Math. Soc., 78 (1972), 1020-1023.

[6] K. Kitano, Invariant subspaces of some non-selfadjoint operators, Tôhoku Math. J., II Ser., 20 (1968), 313-322.

[7] K. Kitano, The growth of the resolvent and hyperinvariant subspaces, Tôhoku Math. J., II Ser., 25 (1973), 317-331.

[8] V. I. MACAEV, A class of completely continuous operators, Doklady Akad. Nauk SSSR, 139 (1961), 548-551.

[9] E. Nordgren, H. Radjavi and P. Rosenthal, On operators with reducing invariant subspaces. (to appear)

[10] P. Rosenthal, Completely reducible operators, Proc. Amer. Math. Soc., 19 (1968), 826830.

[11] T. SAITô, Some remarks on Andô's theorems, Tôhoku Math. J., II Ser., 18 (1966), 404409.

[12] J. T. Schwartz, Subdiagonalization of operators in Hilbert space with compact imaginary part, Comm. Pure Appl. Math., 15 (1962), 159-172.

[13] J. G. StAmpfli, A local spectral theory for operators, J. Functional Analysis, 4 (1969), $1-10$. 
[14] J. G. Stampfir, A local spectral theory for operators, III; Resolvents, spectral sets and similarity, Trans. Amer. Math. Soc., 168 (1972), 133-151.

[15] K. Yosida, Functional Analysis, 2nd Edition, Springer, Berlin-Heidelberg-New York, 1968.

Department of Mathematics

TÔHOKU UNIVERSITY

College of General Education

KaWAUChI, SENDAI, JaPAN 
